\section{Testing the interhemispheric deficit theory of dyslexia using the visual half-field technique} (11)
Quarterly Journal of Experimental Psychology

$1-13$

(C) Experimental Psychology Society 2020 (c) (1) (5)

Article reuse guidelines: sagepub.com/journals-permissions DOI: $10.1177 / 1747021819895472$ qjep.sagepub.com

(SSAGE

\author{
AR Bradshaw $\mathbb{D}$, DVM Bishop $\mathbb{D}$ and ZVJ Woodhead
}

\begin{abstract}
A deficit in interhemispheric transfer has been proposed as a neuropsychological theory of dyslexia. Interactions between the hemispheres during word recognition can be studied using the visual half-field paradigm. The well-established recognition advantage for right visual field (RVF) words over left visual field (LVF) words is thought to reflect the additional processing costs associated with callosal transfer of LVF word representations to the language-specialised left hemisphere. In addition, a further gain in recognition for bilateral presentation of a word has been attributed to cooperative interactions between the hemispheres. These recognition advantages can therefore be seen as behavioural indices of the efficiency of callosal transfer. This study aimed to replicate the finding of an absence of the bilateral advantage in developmental dyslexia, previously reported by Henderson et al. In all, 47 dyslexic and 43 control adult participants were tested, and no significant difference was found in the size of the bilateral advantage between the two groups. Our data did however replicate the previous finding of an increased RVF-LVF difference in dyslexic participants caused by poorer accuracy for LVF words (i.e., a greater LVF cost). This evidence is compatible with the interhemispheric deficit theory of dyslexia, suggesting an impairment in the transfer of visual word information from the right to the left hemisphere during reading.
\end{abstract}

\title{
Keywords
}

Dyslexia; visual half-field paradigm; interhemispheric transfer; corpus callosum; lateralization; reading

Received: 9 April 2019; revised: 4 November 2019; accepted: 24 November 2019

Language and reading require the integration of processing across multiple distributed regions, both within and across the cerebral hemispheres. This requires efficient sharing of sensory inputs and processing outputs between the hemispheres, predominantly mediated by the corpus callosum, the main white matter pathway connecting the two hemispheres. This structure has been proposed to be key in the establishment of lateralised circuits and in mediating interhemispheric transfer (van der Knaap \& van der Ham, 2011). One behavioural index of interhemispheric transfer is superior reading of words presented bilaterally in right and left visual fields compared with words presented unilaterally in the right visual field (RVF), termed the redundant bilateral advantage (RBA). Here, we aimed to replicate a finding by Henderson et al. (2007), who reported a reduced RBA effect on a visual half-field (VHF) task in a dyslexic sample. They argued this was evidence for disrupted interhemispheric transfer during reading in dyslexia.

\section{Models of reading}

As we scan a text or read a centrally fixated word, orthographic information that falls in the left visual field (LVF) will be initially projected to the right hemisphere, with RVF input sent to the left hemisphere (due to decussation of optic fibres at the optic chiasm); at some point, processing of the two VHFs must then be integrated for whole word recognition, requiring callosal transfer. Furthermore, while lower level visual processing is carried out bilaterally, there is evidence that processing of the word form

Department of Experimental Psychology, University of Oxford, Oxford, UK

\section{Corresponding author:}

AR Bradshaw, Department of Speech, Hearing and Phonetic Sciences, University College London, Chandler House, 2 Wakefield Street, London, WCIN IPF, UK.

Email: a.bradshaw@ucl.ac.uk 


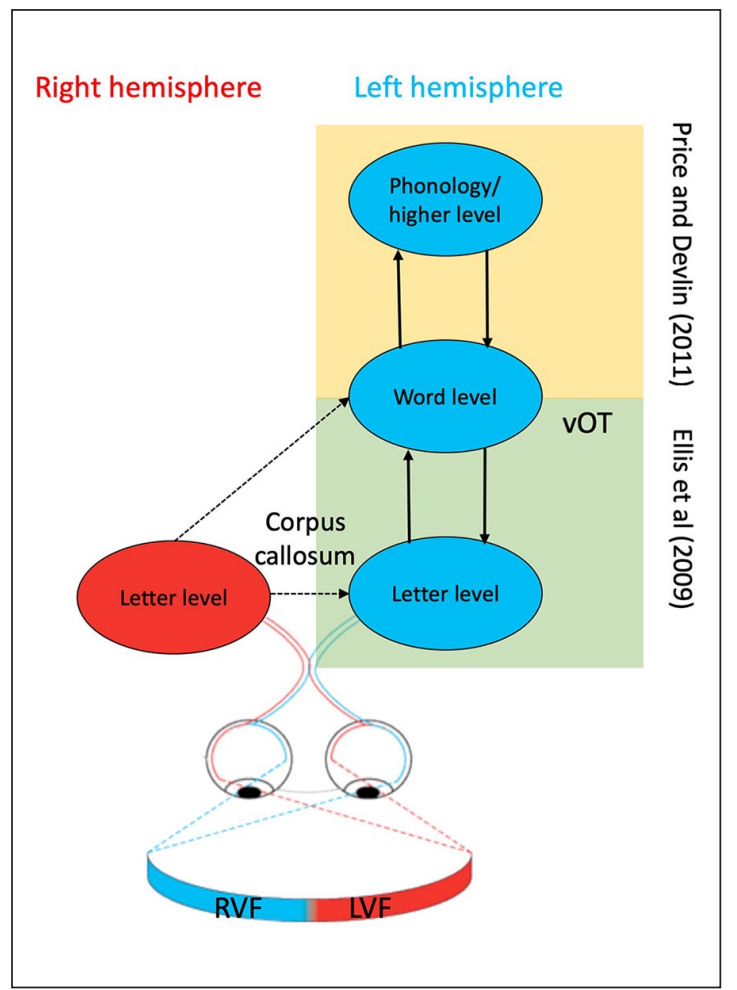

Figure I. Models of hemispheric interactions involved in word recognition. Schematic of the Ellis et al. (2009) and Price and Devlin (20I I) models that propose interactions between different levels in the word recognition process within the left hemisphere. Ellis et al. argue that word recognition is supported by interactions between letter- and word-level representations. Price and Devlin propose that interaction is required between occipital (visual), occipitotemporal (orthographic), and higher-order (e.g., phonological) regions.

becomes left lateralised at around the level of the left ventral occipitotemporal cortex (vOT); this area of cortex in the left hemisphere has been shown to respond with high selectivity to visual word forms presented at any location in the visual field (Cohen et al., 2002).

In their model of word recognition, Ellis et al. (2009) specifically addressed the issue of how the two hemispheres interact. They proposed that efficient recognition of a word is best supported when brain regions at the letter level (left middle/inferior occipital cortex) and word level (left mid fusiform gyrus, that is, vOT) engage in a state of mutual interaction. Ellis et al. argued that this kind of interaction could only occur between regions within the same hemisphere, and not between hemispheres; hence for LVF word input to engage such interactive processing it must first be transferred across the corpus callosum to left vOT. Similarly, Price and Devlin (2011) argued that reading a word requires interactions between occipital, vOT and higher-order phonological areas within the left hemisphere; the latter send top-down predictions to vOT based on stored representations of familiar words. A schematic of these models is given in Figure 1. Overall, visual word form input in the LVF initially projected to the right hemisphere must therefore be transferred to the left hemisphere for such higher order processing to occur. In this way, skilled reading can be seen to rely on efficient interhemispheric interactions and callosal transfer.

\section{Developmental dyslexia and interhemispheric processing}

Dyslexia is a developmental reading disorder that involves severe and persistent difficulties with reading and spelling. It is widely considered to involve a core deficit in phonological processing, in terms of mapping between orthographic inputs and phonological codes, that is, representations of speech sounds (Snowling, 2005). One neuropsychological theory of dyslexia proposes that these reading difficulties emerge as the result of disruption to the transfer of information across the corpus callosum; this is known as the interhemispheric deficit theory of dyslexia (Badzakova-Trajkov et al., 2005; Monaghan \& Shillcock, 2008; Orton, 1927). It has been reported that individuals with dyslexia show deficits on tasks which require interhemispheric transfer, such as bimanual coordination and tactile transfer tasks (Daini et al., 2018; Moore et al., 1995). Structural differences in the corpus callosum have also been reported by multiple studies, although this evidence is not very consistent and likely varies across callosal subregions (for a review, see Paul, 2011). An interesting parallel has been drawn between dyslexia and the types of impairments shown by patients with agenesis of the corpus callosum, who are reported to show similar difficulties with phonological processing such as in rhyming (Banich \& Brown, 2000). This evidence points towards the relevance of abnormalities in interhemispheric transfer to the phonological and reading deficits shown in dyslexia.

\section{The VHF method}

The role of the two hemispheres in word recognition has been investigated behaviourally using the VHF method. This task requires participants to report words that are briefly presented to the right or left visual hemifield. Words presented to the RVF are typically reported faster and more accurately than words presented to the LVF (Hellige, 1993). This RVF-LVF advantage has been attributed to the fact that the representation of a word presented to the LVF/right hemisphere has to be relayed to the left hemisphere via the corpus callosum to be processed by left lateralised language areas, decreasing efficiency of processing (Zaidel et al., 1990). This interpretation is supported by research studying individual differences in language lateralisation. Although the majority of individuals 
show the typical pattern of left lateralisation for language, a minority (around 5\%) show the reverse pattern of greater engagement of the right hemisphere during language processing (Knecht et al., 2000). Critically, there is evidence that these individuals with atypical language lateralisation as measured by fMRI also demonstrate an atypical LVF advantage in VHF tasks, suggesting that this measure is indeed sensitive to an individual's underlying organisation for language (Hunter \& Brysbaert, 2008; van der Haegen et al., 2011).

A further effect that has been reported in VHF tasks is the phenomenon in which simultaneous presentation of identical word stimuli to the two visual hemi-fields results in significantly improved processing (increased accuracy and faster reaction times) compared to when only the RVF is stimulated (Hellige, 1993). This effect has been shown for consonant-vowel-consonant (CVC) syllables (Marks \& Hellige, 2003) and words (Hasbrooke \& Chiarello, 1998; Mohr \& Pulvermüller, 2002), but is absent for pseudo-words (Mohr et al., 2000, 2007). This "redundant bilateral advantage" is not always observed; for instance, Lindell et al. (2003) and Lindell et al. (2005) did not observe it, and it is unclear how far this effect depends on specific tasks or stimuli. Different accounts of the RBA have been proposed, some of which argue that the effect can occur without the need for interhemispheric transfer (e.g., "horse race" models; Miller, 1982) and others which attribute it to cooperative interactions between the hemispheres (Mohr et al., 2007). The finding of an absence of this effect in a split brain patient however strongly suggests it is dependent on an intact corpus callosum and thus interhemispheric communication (Mohr et al., 1994).

\section{Aims of the current study: a replication}

It is of interest then that Henderson et al. (2007) reported a significantly increased RVF-LVF difference and a significantly reduced RBA on the VHF paradigm in dyslexic adults. Compared to controls, dyslexic participants showed both poorer LVF accuracy (resulting in a larger RVF-LVF difference, that is, a greater LVF cost) and a lack of the usual significant difference between accuracy for RVF words and bilaterally presented words. By contrast, when words were presented in top and bottom visual fields dyslexics showed the same redundant advantage as controls, suggesting that their failure to benefit from duplicate presentation relies on the need for interhemispheric transfer between the left and right visual fields. These findings were interpreted as evidence for a dysfunction in callosal transfer in dyslexia, meaning that LVF input is less able to support good recognition alone or to provide a facilitatory effect when presented simultaneously with RVF stimuli. Henderson et al. proposed that the benefit of bilateral stimulation arises because the LVF input reaches left vOT while the RVF input is still being processed; this results in a temporal summation of the inputs to improve recognition. Extending this to the Ellis et al. (2009) model of word recognition, this temporal summation may result in more successful inducement of a state of interaction between letter level and word level areas in the left hemisphere. They suggested that the reduced bilateral advantage in dyslexic participants may reflect slower or delayed interhemispheric transfer that means LVF input is not able to reach the left hemisphere in time to summate with RVF input. The aim of the current study is to replicate these findings of a larger LVF cost and reduced or absent RBA in dyslexic adults relative to typical readers, to address questions as to the processes underlying typical and impaired reading. Furthermore, we aimed to relate the sizes of the bilateral advantage and RVF-LVF difference scores to measures of word and non-word reading ability.

\section{Method}

This study was preregistered as a replication study on Open Science Framework (OSF) prior to commencement of data collection. This registration form along with the anonymised data and analysis scripts can be found on OSF at https://osf.io/4zdhf/.

\section{Participants}

Forty-seven individuals with a diagnosis of dyslexia (mean age: 23.53 years, range: $19-46$ years, 7 left handed) and 43 individuals without any diagnosis of language and/or reading impairment (mean age: 21.87 years, range: 18 -37 years, 2 left handed) were recruited for this study. Participants were recruited predominantly from local institutes of higher education, either via disability services for recruitment of the dyslexic group or via more general advertisements for the control group. Inclusion criteria were English as a first language and an age of 18-50years. Exclusion criteria were significant hearing loss, history of neurological disease, head injury, or epilepsy. Individuals recruited for the dyslexic group all had a confirmed diagnosis of dyslexia, received either from a trained professional (e.g., educational psychologist) while at school $(70.21 \%)$ or from the disability services at their university $(29.87 \%)$. Most had received special help/learning support during their schooling (57.45\%).

Ethical approval for this study was granted by the Medical Sciences Inter-Divisional Research Ethics Committee (IDREC) at the University of Oxford (approval number R56994/RE001). The experiment was undertaken with the understanding and written informed consent of each participant. 
Table I. Mean age and performance of each group on measures of reading and non-verbal ability.

\begin{tabular}{|c|c|c|c|c|}
\hline \multirow[t]{2}{*}{ Measure } & \multicolumn{2}{|c|}{ Controls } & \multicolumn{2}{|c|}{ Dyslexics } \\
\hline & M & $S D$ & M & $S D$ \\
\hline Age (years) & 21.87 & 3.89 & 23.53 & 4.93 \\
\hline TOWRE word reading standard score & 105.19 & 9.45 & 90.28 & 13.87 \\
\hline TOWRE non-word reading standard score & 109.23 & 9.87 & 92.00 & 13.84 \\
\hline TOWRE overall standard score & 109.21 & 10.04 & 89.30 & 15.45 \\
\hline Cattell non-verbal ability (score out of 24) & 21.00 & 2.15 & 19.57 & 2.36 \\
\hline
\end{tabular}

TOWRE: Test of Word Reading Efficiency; VHF: visual half field.

Values for the dyslexic group on these standardised tests are shown for $n=46$ as one dyslexic participant who participated in the VHF task did not complete these.

\section{Materials and method}

Participants were given the Test of Word Reading Efficiency (TOWRE; Torgesen et al., 1999) to obtain scores for word and non-word reading. This test requires participants to read as many words/non-words as they can within a period of $45 \mathrm{~s}$. Participants also completed Tests 1 and 2 of the Cattell Culture Fair Test (Cattell \& Cattell, 1973 ) to give a measure of non-verbal ability. These were administered to all participants except one dyslexic participant whose VHF accuracy measures were nevertheless included in the data analysis. Mean performance on these tasks and mean age for each group are given in Table 1. The dyslexic group demonstrated significantly poorer performance on the TOWRE than controls (Mann-Whitney $U=302.5, p<.001$ ) and also on the Cattell (MannWhitney $U=637.00, p=.005$ ). There was also a significant difference in age between the groups (Mann-Whitney $U=673.00, p=.009)$. In subsequent analyses, we explored whether non-verbal abilities or age may have influenced our results, but this possibility was ruled out.

For the VHF task, the same method and task design as Henderson et al. (2007) was employed, with minor adjustments. Stimuli were 180 five-letter words selected from the CELEX database; these consisted of lists of words combined from across multiple experiments reported in the Henderson et al. paper to increase the number of trials in the task from 120 to 180 . This was motivated by recommendations made by Hunter and Brysbaert (2008) who argued that at least 150 trials are required to yield reliable results with the VHF paradigm. Eighteen practise word stimuli were also used. For the main part of the task, 60 words were allocated to each visual presentation condition: RVF, LVF, or bilateral visual field (BVF) presentation. Within an experimental session, presentation conditions were pseudo-randomly interleaved. Words were rotated around the three conditions to give six different orders of stimulus presentation; administration of these was counterbalanced across participants.

Each word was presented in upper case in Fixedsys font in size 18 point. They were presented on a $36.5 \times 23-\mathrm{cm}$ screen with a resolution of $1024 \times 768$ pixels, at a viewing distance of $60 \mathrm{~cm}$ kept constant by the use of a chin rest.

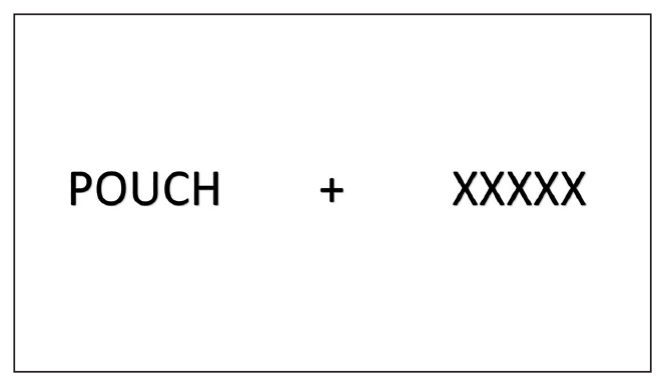

Figure 2. Schematic of stimulus display of target word and contralateral Xs.

The visual angle from the outer edge of the word to the fixation point was around 4.3 degrees, and from the inner edge of the word to fixation around 1.4 degrees; hence, words were presented in parafoveal vision (defined as 1.55 degrees). The task was run using E-prime 1.1 software (Psychology Software Tools, Pittsburgh, PA) using a script identical to that used by Henderson et al. (2007).

A trial began with presentation of a central fixation cross for $400 \mathrm{~ms}$. This was followed by presentation of the word stimulus/stimuli for $60 \mathrm{~ms}$ in one of the three presentation styles. For unilateral presentation trials, a series of $5 \mathrm{Xs}$ were presented in the other visual hemifield to balance the visual presentation (see Figure 2). No mask was used following stimulus presentation, in keeping with the original method of Henderson et al. (2007). Following stimulus presentation, participants were required to use the keyboard to type the previously presented word, and then press enter to start the next trial. Participants were instructed that they could correct any spelling or typing mistakes before pressing enter and to provide their best guess if they were unsure of the word. Scoring allowed for spelling and typing mistakes such that obvious errors were still counted as accurate responses (e.g., THEIF instead of THIEF).

\section{Hypotheses and analysis plan}

All analyses outlined in the following sections were carried out using R (R Core Team, 2019). An Rmd script to perform these analyses can be found on OSF (https://osf.io/4zdhf/). 


\section{Preregistered analyses}

We predicted that, in line with the findings from Henderson et al. (2007), dyslexic adults would show a smaller difference between accuracy in the RVF and BVF conditions compared with typically developed adults; this finding would constitute a successful replication. To test this, we calculated for each individual the difference score between these two conditions (BVF-RVF), referred to as the RBA. We then used an independent groups $t$ test to compare the magnitude of the RBA between the two groups. We predicted that the size of this difference would be significantly smaller in the dyslexic group than in the typically developed group. This analysis differs from the original analysis reported by Henderson et al. (2007). They used a $2 \times 3$ analysis of variance (ANOVA) to compare recognition accuracy between the two groups for RVF, LVF and BVF words and reported a main effect of group, a main effect of condition, and a significant group by condition interaction, indicating that dyslexics showed a smaller bilateral advantage than controls. However, this interaction effect was associated with a very small effect size of $r=.08$, meaning that power calculations based on such an analysis with this effect size gave unrealistically large sample sizes of 200 plus participants. We therefore planned to use this simpler $t$-test analysis with a more focused hypothesis to increase power (see Lazic, 2018, for a discussion).

This simpler analysis gave an effect size of 0.957 calculated from the data reported on by Henderson et al. (2007). $\mathrm{G}^{*}$ Power software was used to calculate that 20 participants would be needed in each group to yield power of 0.9 . However, as effect sizes can be overestimated in published studies particularly if they are under-powered (Button et al., 2013), we aimed to collect data on 30 participants in each group. These data were collected as part of a larger study, and by the end of the data collection period we had data from this task on 47 dyslexics and 43 controls.

\section{Non-preregistered analyses}

We also tested two further hypotheses that were not originally preregistered. First, we predicted that dyslexics would show poorer LVF performance compared with controls; this constitutes an extension of the replication of the findings of Henderson et al. (2007) who reported that dyslexic LVF accuracy but not RVF accuracy was significantly lower than in controls resulting in a greater RVF-LVF difference. Therefore, we tested this using $t$-tests to compare LVF accuracy and RVF accuracy between groups. All $t$-tests were Bonferroni corrected; as three $t$-tests were conducted, a corrected $p$ value of .016 was used. To test more directly for an increased LVF cost in dyslexia, this analysis was followed up by a multi-level mixed models analysis to test for a significant interaction between group and condition. Two random intercept models were compared: a null model with fixed effects of group and condition (with no interaction) and random effects of participant and stimulus, and a full model with the same fixed and random effects but with an interaction between group and condition. A likelihood ratio test was used to obtain a $p$ value for the comparison between the full model and the null model.

Second, we predicted that the size of the RBA and RVFLVF difference scores would correlate with measures of word and non-word reading performance as assessed by the TOWRE. Increased efficiency of interhemispheric transfer would be indexed by a small RVF-LVF difference and large RBA. Thus, assuming efficient interhemispheric transfer is beneficial for reading, we predicted a negative correlation for word and non-word reading scores with RVF-LVF difference scores, but a positive correlation with the size of the RBA. It should be noted that although these advantage measures were most relevant for the current hypothesis, the use of difference scores in analyses has been critiqued due to the unreliability of such difference scores compared to the reliability of the base measures used to calculate them (Thomas \& Zumbo, 2012). The results of these analyses should therefore be interpreted with caution.

Henderson et al. (2007) only included left-handed participants, whereas our sample comprised both right and left-handers, as VHF data were collected as part of a larger study. To rule out handedness as a potential confound in our analyses, we therefore re-ran the group comparisons excluding left-handers.

\section{Results}

\section{Preregistered analysis: replication of comparison of RBA in dyslexics and controls}

Means and standard deviations for each VHF condition can be found for each group in Table 2 along with their mean RBA and RVF-LVF difference scores. Percentage accuracy for each condition is plotted in Figure 3 and given in Table 3 ; these show both the data reported on by Henderson et al. (2007) and the current study. Five controls and one dyslexic participant in our sample failed to demonstrate the typical RVF-LVF advantage (LVF and RVF scores were either identical or greater for LVF than RVF). Handedness could not explain this individual variability in the presence of the RVF-LVF advantage, because all left-handers demonstrated a positive RVF-LVF difference.

RBA scores for each group can be seen in Figure 4. To first confirm that a significant RBA was observed in our data, we used a one-sample $t$ test on the RBA scores of the control group. This confirmed that these scores were significantly different from zero, $t(42)=6.08, p<.001$, and therefore a robust RBA effect was observed in control participants. Our first preregistered analysis of interest was to 
Table 2. Mean accuracy (and SDs) out of 60 on VHF measures for the control and dyslexic groups.

\begin{tabular}{llllll}
\hline Group & LVF accuracy & RVF accuracy & BVF accuracy & RVF-LVF difference & Redundant bilateral advantage \\
\hline Controls & 34.65 & 47.02 & 51.86 & 12.37 & 4.84 \\
& $(S D=10.49)$ & $(S D=7.4 I)$ & $(S D=5.5 \mathrm{I})$ & $(S D=11.39)$ & $(S D=5.21)$ \\
Dyslexics & 27.74 & 43.91 & 47.74 & 16.17 & 3.83 \\
& $(S D=11.64)$ & $(S D=7.16)$ & $(S D=7.12)$ & $(S D=9.45)$ & $(S D=5.85)$ \\
\hline
\end{tabular}

VHF: visual half field; LVF: left visual field; RVF: right visual field; BVF: bilateral visual field.

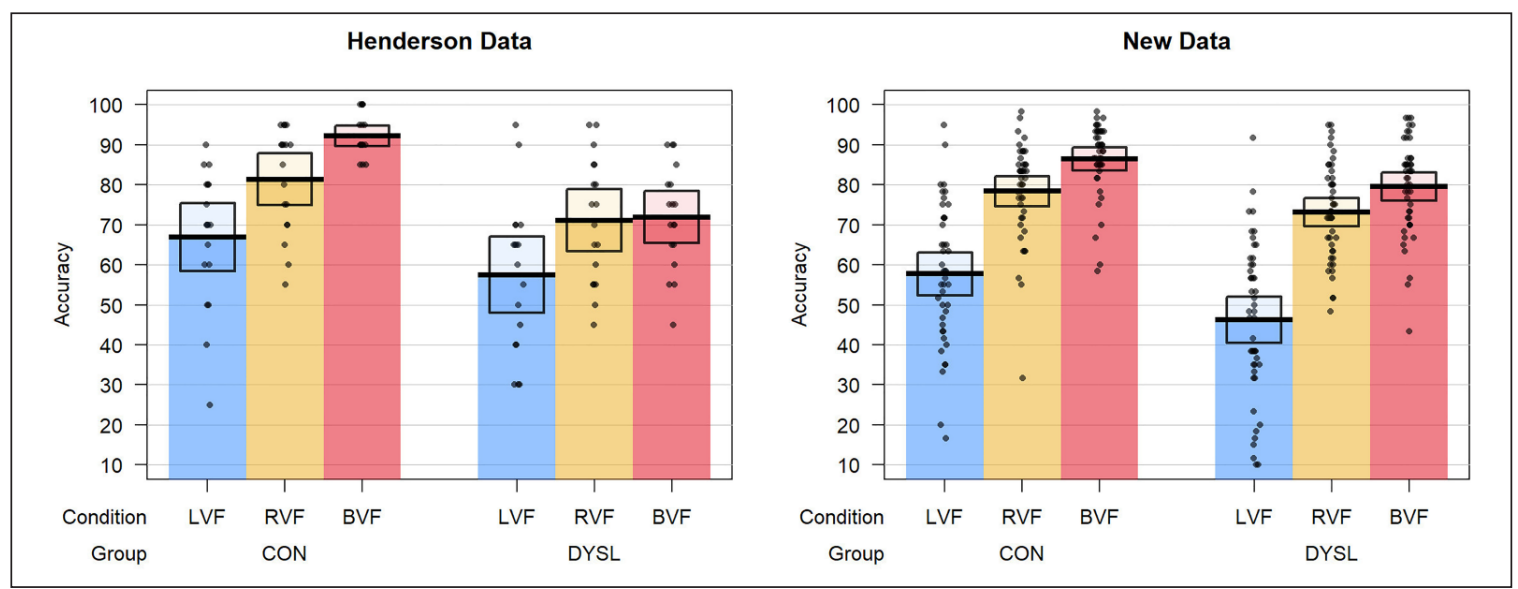

Figure 3. Accuracy data reported from the original study by Henderson et al. (2007) and our study. Plots show individual and mean accuracy scores (as percentage accuracy) for each visual hemifield condition in the two groups. Boxes indicate $95 \%$ confidence intervals. Note that data for the Henderson study is given for $n=18$ in each group, as data for the full groups reported on in the paper were not available. CON: control group; DYSL: dyslexia group.

Table 3. Percentage accuracy in the two groups across the three visual half-field conditions for Henderson et al. and the current study.

\begin{tabular}{lccccccc}
\hline \multirow{2}{*}{ Study } & \multicolumn{3}{l}{ Dyslexics } & & \multicolumn{3}{l}{ Controls } \\
\cline { 2 - 4 } \cline { 7 - 8 } & LVF & RVF & BVF & & LVF & RVF & BVF \\
\hline $\begin{array}{l}\text { Henderson et al. } \\
(n=44)\end{array}$ & $53 \%$ & $72.7 \%$ & $72 \%$ & $68 \%$ & $80.7 \%$ & $92 \%$ \\
$\begin{array}{l}\text { Bradshaw et al. } \\
(n=90)\end{array}$ & $46 \%$ & $73 \%$ & $79.6 \%$ & $57.7 \%$ & $78.4 \%$ & $86.4 \%$ \\
\hline
\end{tabular}

LVF RVF: right visual field; BVF: bilateral visual field.

then compare the RBA scores between control and dyslexic groups. An independent samples $t$ test found no significant difference in the size of the RBA between groups, $t(88)=.859, p=.393$. Thus, we did not replicate the finding of a reduced bilateral advantage in a dyslexic sample compared to controls. This result was unchanged if only righthanders were included in the analysis. As neither age nor Cattell score correlated with RBA scores, group differences in these variables are unlikely to have affected this result.

\section{Non-preregistered exploratory analyses}

We conducted an additional non-preregistered analysis to replicate the second analysis done by Henderson et al.
(2007), comparing LVF and RVF accuracy between groups, with the hypothesis that the former and not the latter will be significantly different. An independent $t$ test found a significant difference between groups for the LVF condition, with dyslexics showing lower accuracy than controls, $t(88)=2.95, p=.004, d=0.62$. As RVF accuracy data were found to be non-normal, this was compared between groups by means of a Mann-Whitney $U$ test. This did not find a significant difference between groups at the Bonferroni corrected $p$ value (Mann-Whitney $U=720.5$, $p=.019, r=.247)$. Therefore, we replicated Henderson et al.'s (2007) finding of poorer performance of dyslexics for LVF presented words, that is, an increased LVF cost.

To test more directly for an increased LVF cost in dyslexia, we used a multi-level mixed model analysis to test for a significant interaction between group (control vs. dyslexic) and condition (RVF vs. LVF) on accuracy scores. A likelihood ratio test was used to compare a null model with fixed effects of group and condition (with no interaction) and random effects of participant and stimulus, with a full model with the same fixed and random effects but with an interaction between group and condition. This found that the full model provided a better fit to the data, $\chi^{2}(1)=18.50, p<.001$. This result was unchanged when excluding left-handed participants. Thus, a significant interaction was found between condition and group in 


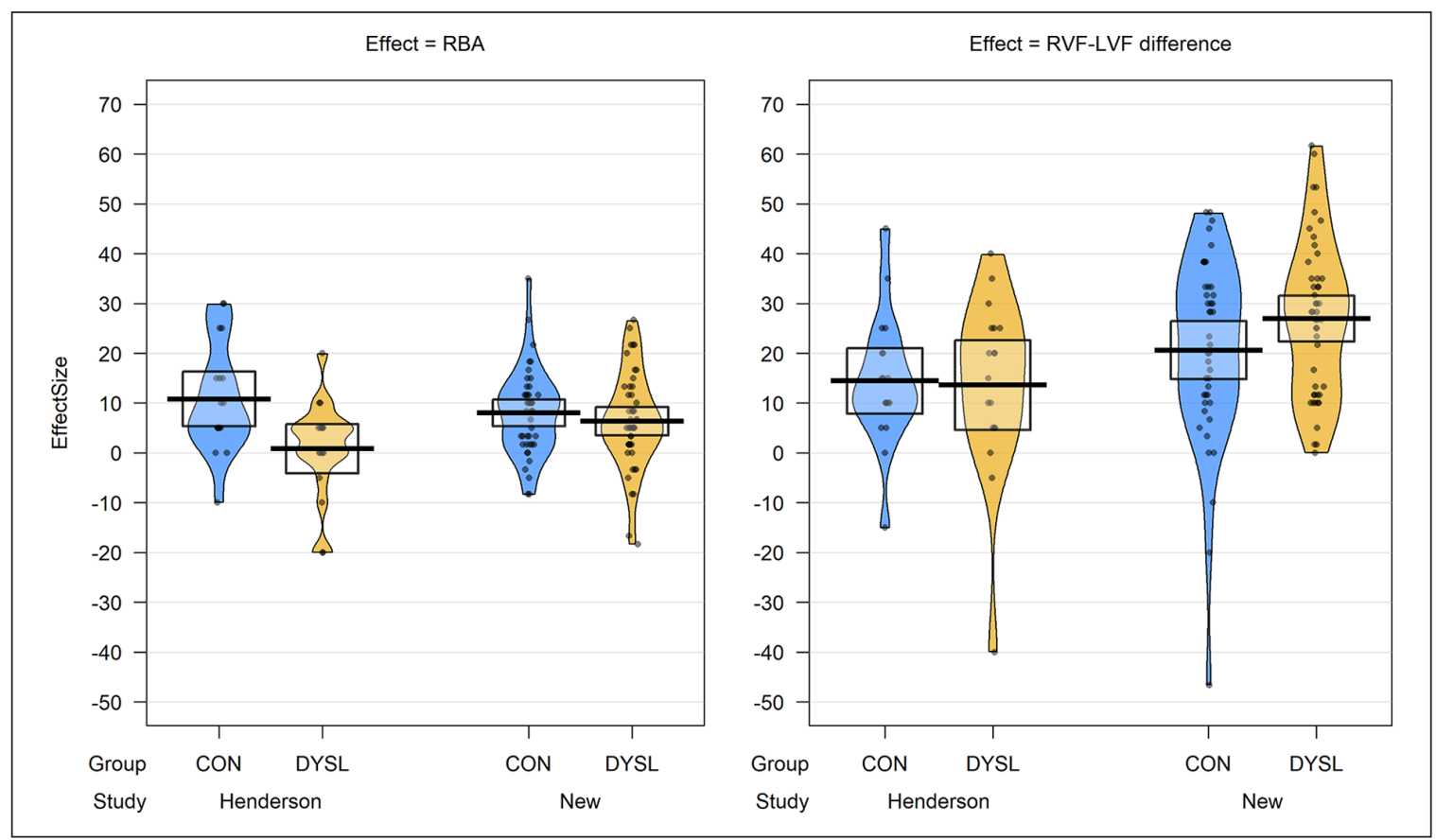

Figure 4. Pirate plots showing mean and individual data points for bilateral advantage and RVF-LVF difference scores in the two groups, from the original Henderson et al. study and the current study. Shaded boxes indicate $95 \%$ confidence intervals. Note that data for the Henderson study is given for $n=18$ in each group, as data for the full groups reported on in the paper were not available. CON: control group; DYSL: dyslexia group.

which the dyslexic group demonstrated a greater difference between LVF and RVF conditions than the control group. As can be seen in Figure 3, this larger RVF-LVF difference was driven by poorer performance in the LVF condition, rather than better performance for RVF words; this thus supports the idea of an increased LVF cost in dyslexia.

Furthermore, we ran exploratory analyses to investigate the relationship between TOWRE word and non-word reading standard scores and the RBA and RVF-LVF difference scores. Pearson's correlations found no significant relationship between TOWRE word reading standard scores and either the RBA $(r=.002, p=.984)$ or RVF-LVF difference scores $(r=-.102, p=.341)$. Similarly, no significant correlations were found between TOWRE non-word reading standard scores and either the RBA $(r=-.029$, $p=.784)$ or RVF-LVF difference scores $(r=-.072$, $p=.503)$.

\section{Discussion}

\section{Summary of results}

This study aimed to use the VHF paradigm to replicate the finding of a reduced RBA in dyslexic adults compared with controls. Although a RBA was observed, we failed to replicate the significant difference between the size of this advantage in dyslexics and controls. We did however replicate the finding of an increased RVF-LVF difference in dyslexics compared to controls. This was driven by significantly worse performance of the dyslexics on the LVF condition as previously reported in a right-handed only sample by Henderson et al. (2007), and so could be more accurately termed an increased LVF cost in dyslexia (we will use this term for the remainder of the "Discussion"). Therefore, our results provide partial support for the hypothesis of impaired interhemispheric transfer in dyslexia, but suggest that the nature of this impairment does not necessarily interfere with the bilateral advantage. Correlation analyses found that neither word nor non-word reading scores were significantly correlated with either the RVF-LVF difference or bilateral advantage scores.

\section{Poorer LVF accuracy in dyslexia}

The main pattern observed in the performance of the dyslexic group was a particular difficulty with words presented in the LVF. In contrast, accuracy for RVF words did not differ from that of controls. This replicated Henderson et al.'s (2007) finding, who also reported an increased LVF cost associated with dyslexia in their sample of right-handers. This pattern of a specific difficulty with LVF words is consistent with the interhemispheric deficit theory of dyslexia. According to models of reading (Ellis et al., 2009; Price \& Devlin, 2011), accurate recognition of LVF words 
requires transfer of this visual input across the corpus callosum to access left lateralised processing circuits involving reciprocal interactions between the letter, word, and phonological levels. Impairment in the transfer of this LVF input across the corpus callosum would therefore prevent access to this more efficient mode of processing, meaning word recognition has to rely on more basic and perhaps more serial letter-by-letter processes in the right hemisphere (Ellis et al., 2009).

The idea that dyslexic readers struggle particularly with reading word forms presented in their LVF is also supported by eye tracking research. Kelly et al. (2004) studied eye movements in dyslexics and controls during reading of sentences and found that dyslexics showed (1) more fixations to the left (i.e., at the beginning) of words and (ii) shorter fixation durations when fixating on the beginning of words than their centre. They interpreted this bias for more leftward fixations as a strategy employed by dyslexic readers to ensure most letter information in a word is directly projected to the left hemisphere to avoid the need for callosal transfer. Such patterns in eye movements could constitute a compensatory mechanism for the disrupted transfer of LVF input to the left hemisphere as suggested by our findings.

The LVF cost in dyslexia may also be interpreted within the SERIOL (sequential encoding regulated by inputs to oscillations within letter units) model of word reading (Whitney, 2001; Whitney \& Cornelissen, 2005). This computation model aims to specify how word recognition occurs, from early retinotopic visual processing, via abstract feature, letter and bigram representations, to lexical word form representations. Of particular relevance to our study is the way in which the early retinotopic activation is transformed into serial abstract letter encoding. The retinotopic activation is characterised by an acuity gradient, where the most central retinal locations are activated most strongly. To attain the left-to-right serial order of the abstract lexical representation, the acuity gradient in the LVF/right hemisphere has to be reversed. The model achieves this gradient inversion by strong left-to-right lateral inhibition in the right hemisphere. Whitney and Cornelissen (2005) argue that this inversion is a readingspecific skill, and that a failure to acquire it may result in developmental dyslexia. This mechanistic explanation of dyslexia would predict that participants with dyslexia would perform more poorly on reading words in the LVF, as we have observed here; however, a study of letter identification with consonant triads presented to the left or right visual fields failed to find the three-way interaction between hemifield, letter position, and group that would be predicted by the SERIOL model (Callens et al., 2013). The study by Callens et al. had only 20 participants per group and has not (to our knowledge) been replicated; but taken at face value, their results suggested that inversion of the acuity gradient was not the origin of reading problems in dyslexia, and that the LVF cost may occur at the word level and not for letter reporting alone.

These results may also be interpreted in the light of studies showing impaired visual attention in dyslexia. There is a substantial literature on attention impairments in dyslexia (e.g., Bosse et al., 2007; Hari \& Renvall, 2001; Valdois et al., 2004; Vidyasagar \& Pammer, 1999). Some studies indicate that the visual attention deficit may be asymmetrical, affecting the LVF more than the RVF. For example, Hari and colleagues describe left hemifield "minineglect" in adults with dyslexia, who show LVF deficits in non-verbal tasks such as temporal order decisions or line motion illusions (Hari, 2001). Similarly, Facoetti and colleagues reported mild LVF inattention and RVF overdistractability in children with dyslexia (Facoetti et al., 2001; Facoetti \& Molteni, 2001; Facoetti \& Turatto, 2000). It has been speculated that these results may indicate a minor deficit in the right parietal lobe in dyslexia (Hari \& Renvall, 2001). This asymmetric visual attention theory could provide an alternative explanation for the LVF cost observed here, as it would predict that words presented in the LVF would be less attention grabbing, and more prone to distraction from the "XXXXX" stimulus presented in the contralateral (right) visual field.

Interestingly, there is evidence that dyslexic participants may show the reverse pattern of heightened hemifield preferences when the task is right lateralised. Daini et al. (2018) measured performance of dyslexic children on an orientation matching task where a picture pair with either matching or discrepant orientations was presented to either the right or left visual field. In control participants, this task was found to involve an LVF advantage, indicative of right lateralisation. Dyslexic participants demonstrated an increased LVF-RVF difference due to a specific difficulty with RVF stimuli, consistent with an impairment in the transfer of RVF input to the specialised right hemisphere. This increased "RVF cost" represents a parallel to the increased LVF cost reported in the current paper. Furthermore, such a pattern has also been reported in split brain patients, who showed a larger RVF cost for this orientation matching task (Corballis et al., 2010). It is worth noting that neither the SERIOL model nor the visual attentional account can explain the RVF cost in dyslexia for this non-verbal task. Together with the present findings, this therefore suggests that dyslexia involves a general impairment in the transfer of visual information across the corpus callosum, regardless of the required direction or the stimulus domain.

To integrate these two sets of findings, it would be of interest to measure behavioural asymmetries for both right- and left-lateralised tasks within the same participants (both typical and dyslexic) to establish that these demonstrate correspondence at the individual level. Specifically, one would predict that individuals who demonstrate a larger LVF cost for word stimuli as in the current 
method should also show a larger RVF cost for non-verbal stimuli as in the method used by Daini et al. This would support the idea that these measures from the two types of task are sensitive to the same underlying construct, assumed to be efficiency of interhemispheric transfer.

\section{Failure to replicate a reduced RBA in dyslexia}

More puzzling however is why such a deficit in interhemispheric transfer suggested by poorer LVF performance was not apparent in performance for bilateral presentation in the dyslexic group. When interpreting their findings, Henderson et al. (2007) suggested that the absence of the bilateral advantage in dyslexics may be due to issues in the timing of interhemispheric transfer; that is, the LVF input may not reach the left hemisphere in time to summate with the RVF input and "boost" processing. An alternative view would be that interhemispheric transfer is not slower but instead degraded such that the representation of the LVF word form transmitted to the left hemisphere is of poor quality. Such a scenario was in fact induced in typical readers in an experiment in the Henderson et al. (2007) paper. Here, RVF input was presented simultaneously with a degraded version of the same word in the LVF in the form of a word fragment consisting of the first and last letters of the word (e.g., c-n for coin). This bilateral presentation was sufficient to induce a bilateral advantage in the controls; however, lateralised presentation of the word fragment alone did not allow for its successful recognition. In light of our findings, one might suggest that the bilateral advantage demonstrated in our dyslexic group could be supported by a similar process of integration of degraded LVF input (due to faulty interhemispheric transfer rather than stimulus manipulation) with full-word RVF input. That is, this LVF input can arrive in time to summate with the RVF input and result in a bilateral advantage, but is degraded in quality and so insufficient by itself to support accurate word recognition (resulting in an increased LVF cost).

These two models of the nature of the deficit in interhemispheric transfer in dyslexia should be further tested against each other. The latency of interhemispheric transfer times (IHTTs) can be measured using event-related potentials (ERPs) to record the delay between a particular ERP component evoked in the contralateral hemisphere by a word stimulus and that evoked in the ipsilateral hemisphere. This method has been used to demonstrate a directional asymmetry in controls in which transfer of verbal material is faster from the right hemisphere to the left than vice versa (Brown et al., 1994). Using this method, Martin et al. (2007) reported an absence of this asymmetry in dyslexic participants, who instead showed balanced latencies for transfer of visual word information in both directions. However, while overall IHTTs were reported as "marginally shorter" in controls than dyslexics, this did not reach statistical significance in their small sample (10 participants per group) and therefore should not be interpreted as evidence for slower callosal transfer in dyslexia.

\section{Methodological differences between the studies}

The failure to replicate the group difference in the bilateral advantage reported by Henderson et al. (2007) could simply indicate that this previous finding was a false positive which our higher powered study was able to avoid. The relatively small number of participants in the Henderson et al. study suggests that sampling error could well be responsible for the discrepancy in findings. Nevertheless, it is important to examine any potential methodological differences between our study and theirs. Figure 3 and Table 3 reveal that performance of our control participants across the three conditions was slightly reduced compared to that of the controls reported on by Henderson et al.; furthermore, our bilateral advantage was slightly smaller (around $8 \%$ vs. 12\%). This lower accuracy is puzzling given that the same task was administered using the same script to very similar groups of participants. The key difference between our procedures was in the number of trials, with our study increasing this from 120 to 180 in line with recommendations from Hunter and Brysbaert (2008). This increase in the number of measurements taken would have further increased the power of our design to detect group differences.

The make-up of our dyslexic and control groups was similar to those of the original study in age, student status, and performance on the same measure of reading and nonword reading ability. Our data did however show a higher level of individual variability in accuracy scores than that reported by Henderson et al. (2007), with higher standard errors for each condition in both groups. VHF data are often characterised by a high level of individual variability, which has led many to argue that behavioural methods are too noisy to provide reliable measures (Hunter \& Brysbaert, 2008; Voyer, 1998). Despite this, however, a recent study demonstrated that the majority of effects at the group level reported in the VHF literature do replicate in well powered studies (Brederoo et al., 2019). This suggests that VHF paradigms do yield robust and reliable group effects, despite their potential for high individual variability.

The current study did deviate from the methods of the original Henderson et al. study in its inclusion of lefthanded participants. However, removing these left-handed participants did not affect the outcome of any of the analyses, and therefore this difference cannot explain the failed replication. It should be noted that in our sample there was no correspondence between handedness and the presence of an RVF-LVF advantage: In fact, all participants with an LVF advantage were right handed. 
The experimental task used by Henderson et al. does deviate from standard design in VHF paradigms in a number of ways. Typically, VHF tasks require participants to verbally report the presented word, thus allowing for reaction times to be measured as well as accuracy. In contrast, the current task required participants to type the word after presentation in a non-timed fashion. This lack of a response time measure seems somewhat counterintuitive with regard to the theory being tested, as one might assume that the effects of inefficient callosal transfer would be most evident in slower response times. However, other studies of the bilateral advantage have reported this effect for both reaction time and accuracy measures (Mohr et al., 2007; Mohr \& Pulvermüller, 2002). Furthermore, a very short stimulus presentation of $60 \mathrm{~ms}$ was used, much shorter than the $150 \mathrm{~ms}$ presentation reported as standard by Hunter and Brysbaert (2008). Other studies with this paradigm have also used picture stimuli rather than written word stimuli, which could make the task easier for dyslexic participants (Hunter \& Brysbaert, 2008). Given that this was a replication study, these design parameters were kept identical to the original study, but future work might consider systematically assessing the effects of such parameters.

\section{Limitations of the study}

One key criticism of the methodological design of the task used by Henderson et al. and the current study is the lack of direct control over the location of participants' eye fixation locations during the tasks. Concerns over the extent to which participants adhere to instructions to fixate centrally have been raised; indeed when eye movements are monitored with eye-tracking, it is often reported that participants fail to maintain central fixation (Jordan et al., 1998; van der Haegen et al., 2011). This is a concern for a paradigm which fundamentally relies on the assumption of central fixation and thus isolated presentation of stimuli to each hemisphere. However, others have reported more reassuring findings that such deviations from central fixation do not in practice affect measurement of key effects. The addition of eye-position contingent stimulus presentation has been reported to have no effect on the RVF-LVF advantage (Jordan et al., 1998) or on the related optimal viewing position curve (van der Haegen et al., 2010), and importantly does not improve correspondence with laterality measurements from fMRI (van der Haegen et al., 2011). To our knowledge, however, no study has used eye-tracking when measuring the RBA effect; this would be useful to validate the presence of the effect when fixation location is controlled.

A further potential criticism of the current design relates to the presentation of strings of Xs to the VHF contralateral to the target stimulus on unilateral trials. This is recommended for use in VHF methods so as to visually balance the stimulus array to discourage spontaneous eyemovements towards the target (Hunter \& Brysbaert, 2008). However, others have suggested that such contralateral perceptual distractors may interfere with processing of the target and so impair recognition performance (Chu \& Meltzer, 2019; Fernandino et al., 2007). Unilateral trials may thus require greater attentional resources to selectively attend to the target word in the face of such interference. Viewed in this way, the so called RBA observed when comparing "unilateral" and redundant bilateral trials could in fact reflect a unilateral disadvantage. It is possible therefore that differences in the findings between the current study and that of Henderson et al. could be due to differences in the extent of attentional difficulties in the two samples. It should be noted, however, that the bilateral advantage effect has been demonstrated in a number of studies that did not use contralateral Xs during unilateral trials (Hasbrooke \& Chiarello, 1998; Mohr et al., 2000, 2007; Mohr \& Pulvermüller, 2002).

\section{Future directions}

To move forward, the interhemispheric deficit hypothesis of dyslexia needs clarification as to what aspect of interhemispheric communication is affected. The corpus callosum is a heterogeneous structure with distinct subregions that are involved in the transfer of different types of information at different levels of processing. For this theory to develop, it will be important for it to take into account what is known about the topography of the corpus callosum. Rather than assuming a global deficit in interhemispheric transfer, dyslexia could involve dysfunction in specific callosal segment(s) leading to disruption to the transfer of specific types of information. Specifying such details would enable more effective testing of the predictions of the theory, such that different aspects of interhemispheric transfer beyond that of visual information can be probed in dyslexia in the context of falsifiable predictions.

Such predictions could be tested using a task developed by Banich and Belger (1990), in which participants make match/mismatch decisions that require comparing across two items. Stimulus presentation is manipulated such that there is either a need for interhemispheric transfer (acrosshemisphere trials, that is, one item presented to each hemisphere) or no need (within-hemisphere trials, that is, both items presented to the same hemisphere). One can then compare accuracy or speed of performance between these types of trials to determine which mode of processing is more advantageous. It is generally found that within-hemisphere processing is more advantageous for simple tasks (e.g., perceptual letter matching, A and A), whereas performance is better for across-hemisphere trials for complex tasks (e.g., identity letter matching, A and a) (Hughes et al., 2016; Weissman \& Banich, 2000). It is proposed that 
the benefits of increased computational power associated with cooperative interhemispheric processing outweigh the costs of requiring interhemispheric transfer only when the task is sufficiently complex. Assuming a deficit in interhemispheric transfer in dyslexia, one might predict that dyslexic participants would show better performance for within-hemisphere trials than between-hemisphere trials regardless of task complexity. This paradigm would further allow for testing of whether such a theorised pattern of performance in dyslexia is domain specific (e.g., specific to written word forms/letters) or seen across different types of stimuli (e.g., digits, pictures), to refine the interhemispheric deficit hypothesis. Indeed, this paradigm was used in the study by Daini et al. (2018) to demonstrate an increased RVF cost for non-verbal picture stimuli in dyslexic participants as previously discussed, suggesting the hypothesised impairment in interhemispheric transfer may be domain general.

Finally, one must consider that rather than being a cause of dyslexia, any theorised differences in interhemispheric transfer could instead be a consequence of poor acquisition of reading skill. In a study of adult illiterates, learning to read as an adult was found to be associated with increased white matter in the splenium of the corpus callosum (Carreiras et al., 2009), suggesting that acquisition of reading skill may itself drive development of more efficient interhemispheric transfer. Thus, although the present results provide support for an association between weaker interhemispheric transfer and dyslexia, they cannot shed light on the direction of causality within this relationship. Further work on the interhemispheric deficit hypothesis of dyslexia should seek to address this.

\section{Summary and conclusion}

This study highlights the importance of replication studies to ensure that a significant effect frequently cited in the literature is a true effect. We found that while an increased LVF cost in dyslexic participants does provide support for the interhemispheric deficit theory of dyslexia, our failure to replicate a reduced RBA in dyslexia does not. This pattern may, however, reflect the type of disruption to interhemispheric transfer in dyslexia, being more consistent with a model of degraded transfer of LVF word input to the left hemisphere than slow callosal transfer. This proposal should be further investigated by studies explicitly designed to test the predictions of the two models. Further work is also needed to refine the interhemispheric deficit theory of dyslexia, to generate more specific testable hypotheses as to which aspects of interhemispheric transfer are affected.

\section{Acknowledgements}

The authors would like to thank Dr. Lisa Henderson for her willingness to share experiment materials and data from the original study. The authors would also like to thank Miss Leanne Smith for her help with data collection, and Dr. Paul Thompson for his help with the multi-level modelling analysis.

\section{Declaration of conflicting interests}

The author(s) declared no potential conflicts of interest with respect to the research, authorship, and/or publication of this article.

\section{Funding}

The author(s) disclosed receipt of the following financial support for the research, authorship, and/or publication of this article: This work was supported by an advanced grant awarded by the European Research Council (project 694189-Cerebral Asymmetry: New directions in Correlates and Etiology [CANDICE]). Dorothy Bishop is funded by programme grant 082498/Z/07/Z from the Wellcome Trust.

\section{ORCID iDs}

AR Bradshaw (iD) https://orcid.org/0000-0003-0138-5945

DVM Bishop (iD https://orcid.org/0000-0002-2448-4033

\section{Open practices}

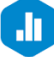

All analysis scripts and anonymised data are available on Open Science Framework (https://osf.io/4zdhf/).

\section{References}

Badzakova-Trajkov, G., Hamm, J. P., \& Waldie, K. E. (2005). The effects of redundant stimuli on visuospatial processing in developmental dyslexia. Neuropsychologia, 43(3), 473-478. https://doi.org/10.1016/j.neuropsychologia.2004.06.016

Banich, M. T., \& Belger, A. (1990). Interhemispheric interaction: How do the hemispheres divide and conquer a task? Cortex, 26(1), 77-94. https://doi.org/10.1016/S00109452(13)80076-7

Banich, M. T., \& Brown, W. S. (2000). A life-span perspective on interaction between the cerebral hemispheres. Developmental Neuropsychology, 18(1), 1-10. https://doi. org/10.1207/S15326942DN1801_1

Bosse, M. L., Tainturier, M. J., \& Valdois, S. (2007). Developmental dyslexia: The visual attention span deficit hypothesis. Cognition, 104(2), 198-230. https://doi. org/10.1016/j.cognition.2006.05.009

Brederoo, S. G., Nieuwenstein, M. R., Cornelissen, F. W., \& Lorist, M. M. (2019). Reproducibility of visual-field asymmetries: Nine replication studies investigating lateralization of visual information processing. Cortex, 111, 100-126. https://doi.org/10.1016/j.cortex.2018.10.021

Brown, W. S., Larson, E. B., \& Jeeves, M. A. (1994). Directional asymmetries in interhemispheric transmission time: Evidence from visual evoked potentials. Neuropsychologia, 32(4), 439-448. https://doi.org/10.1016/0028-3932(94) 90089-2

Button, K. S., Ioannidis, J. P. A., Mokrysz, C., Nosek, B. A., Flint, J., Robinson, E. S. J., \& Munafò, M. R. (2013). Power 
failure: Why small sample size undermines the reliability of neuroscience. Nature Reviews Neuroscience, 14(5), 365376. https://doi.org/10.1038/nrn3475

Callens, M., Whitney, C., Tops, W., \& Brysbaert, M. (2013). No deficiency in left-to-right processing of words in dyslexia but evidence for enhanced visual crowding. Quarterly Journal of Experimental Psychology, 66(9), 1803-1817. https://doi.org/10.1080/17470218.2013.766898

Carreiras, M., Seghier, M. L., Baquero, S., Estévez, A., Lozano, A., Devlin, J. T., \& Price, C. J. (2009). An anatomical signature for literacy. Nature, 461(7266), 983-986. https://doi. org/10.1038/nature08461

Cattell, R. B., \& Cattell, A. K. S. (1973). Handbook for the Culture Fair Intelligence Test: Scale 2. Institute for Personality and Ability Testing.

Chu, R. K., \& Meltzer, J. A. (2019). Interhemispheric connectivity during lateralized lexical decision. Human Brain Mapping, 40(3), 818-832. https://doi.org/10.1002/hbm.24414

Cohen, L., Lehéricy, S., Chochon, F., Lemer, C., Rivaud, S., $\&$ Dehaene, S. (2002). Language-specific tuning of visual cortex? Functional properties of the Visual Word Form Area. Brain, 125(5), 1054-1069. https://doi.org/https://doi. org/10.1093/brain/awf094

Corballis, M. C., Birse, K., Paggi, A., Manzoni, T., Pierpaoli, C., $\&$ Fabri, M. (2010). Mirror-image discrimination and reversal in the disconnected hemispheres. Neuropsychologia, 48(6), 1664-1669. https://doi.org/10.1016/j.neuropsychologia.2010.02.011

Daini, R., De Fabritiis, P., Ginocchio, C., Lenti, C., Lentini, C. M., Marzorati, D., \& Lorusso, M. L. (2018). Revisiting strephosymbolie: The connection between interhemispheric transfer and developmental dyslexia. Brain Sciences, 8(4), Article 67. https://doi.org/10.3390/brainsci8040067

Ellis, A. W., Ferreira, R., Cathles-Hagan, P., Holt, K., Jarvis, L., \& Barca, L. (2009). Word learning and the cerebral hemispheres: From serial to parallel processing of written words. Philosophical Transactions of the Royal Society B: Biological Sciences, 364(1536), 3675-3696. https://doi.org/ https://doi.org/10.1098/rstb.2009.0187

Facoetti, A., \& Molteni, M. (2001). The gradient of visual attention in developmental dyslexia. Neuropsychologia, 39(4), 352-357. https://doi.org/10.1016/S0028-3932(00)00138-X

Facoetti, A., \& Turatto, M. (2000). Asymmetrical visual fields distribution of attention in dyslexic children: A neuropsychological study. Neuroscience Letters, 290(3), 216-218. https://doi.org/10.1016/S0304-3940(00)01354-9

Facoetti, A., Turatto, M., Lorusso, M. L., \& Mascetti, G. G. (2001). Orienting of visual attention in dyslexia: Evidence for asymmetric hemispheric control of attention. Experimental Brain Research, 138(1), 46-53. https://doi. org/10.1007/s002210100700

Fernandino, L., Iacoboni, M., \& Zaidel, E. (2007). The effects of bilateral presentations on lateralized lexical decision. Brain and Cognition, 64(1), 60-67. https://doi.org/10.1016/J. BANDC.2006.11.004

Hari, R. (2001). Left minineglect in dyslexic adults. Brain, 124(7), 1373-1380. https://doi.org/10.1093/brain/124.7.1373

Hari, R., \& Renvall, H. (2001). Impaired processing of rapid stimulus sequences in dyslexia. Trends in Cognitive
Sciences, 5(12), 525-532. https://doi.org/10.1016/S13646613(00)01801-5

Hasbrooke, R.E., \& Chiarello, C.(1998). Bihemispheric processing of redundant bilateral lexical information. Neuropsychology, 12(1), 78-94. https://doi.org/10.1037//0894-4105.12.1.78

Hellige, J. B. (1993). Perspectives in cognitive neuroscience. Hemispheric asymmetry: What's right and what's left. Harvard University Press.

Henderson, L., Barca, L., \& Ellis, A. W. (2007). Interhemispheric cooperation and non-cooperation during word recognition: Evidence for callosal transfer dysfunction in dyslexic adults. Brain and Language, 103(3), 276-291. https://doi. org/https://doi.org/10.1016/j.bandl.2007.04.009

Hughes, A. J., Upshaw, J. N., Macaulay, G. M., \& Rutherford, B. J. (2016). Enhancing the ecological validity of tests of lateralization and hemispheric interaction: Evidence from fixated displays of letters or symbols of varying complexity. Brain and Cognition, 106, 1-12. https://doi.org/10.1016/j. bandc.2016.04.012

Hunter, Z. R., \& Brysbaert, M. (2008). Visual half-field experiments are a good measure of cerebral language dominance if used properly: Evidence from fMRI. Neuropsychologia, 46(1), 316-325. https://doi.org/10.1016/j.neuropsychologia.2007.07.007

Jordan, T. R., Patching, G. R., \& Milner, A. D. (1998). Central fixations are inadequately controlled by instructions alone: Implications for studying cerebral asymmetry. Quarterly Journal of Experimental Psychology Section A: Human Experimental Psychology, 51(2), 371-391. https://doi. org/10.1080/713755764

Kelly, M. L., Jones, M. W., McDonald, S. A., \& Shillcock, R. C. (2004). Dyslexics' eye fixations may accommodate to hemispheric desynchronization. NeuroReport, 15(17), 26292632. https://doi.org/10.1097/00001756-200412030-00014

Knecht, S., Dräger, B., Deppe, M., Bobe, L., Lohmann, H., Flöel, A., . . .Henningsen, H. (2000). Handedness and hemispheric language dominance in healthy humans. Brain, 123(12), 2512-2518. https://doi.org/10.1093/brain/123.12.2512

Lazic, S. E. (2018). Four simple ways to increase power without increasing the sample size. Laboratory Animals, 52, 621-629. doi:10.1177/0023677218767478

Lindell, A. K., Nicholls, M. E. R., \& Castles, A. E. (2003). The effect of orthographic uniqueness and deviation points on lexical decisions: Evidence from unilateral and bilateral-redundant presentations. Quarterly Journal of Experimental Psychology Section A: Human Experimental Psychology, 56(2), 287-307. https://doi. org/10.1080/02724980244000341

Lindell, A. K., Nicholls, M. E. R., Kwantes, P. J., \& Castles, A. (2005). Sequential processing in hemispheric word recognition: The impact of initial letter discriminability on the OUP naming effect. Brain and Language, 93(2), 160-172. https://doi.org/10.1016/j.bandl.2004.09.006

Marks, N. L., \& Hellige, J. B. (2003). Interhemispheric interaction in bilateral redundancy gain: Effects of stimulus format. Neuropsychology, 17(4), 578-593. https://doi. org/10.1037/0894-4105.17.4.578

Martin, C. D., Thierry, G., Démonet, J. F., Roberts, M., \& Nazir, T. (2007). ERP evidence for the split fovea theory. Brain 
Research, 1185, 212-220. https://doi.org/10.1016/j.brainres.2007.09.049

Miller, J. (1982). Divided attention: Evidence for coactivation with redundant signals. Cognitive Psychology, 14(2), 247279. https://doi.org/10.1016/0010-0285(82)90010-X

Mohr, B., Endrass, T., Hauk, O., \& Pulvermüller, F. (2007). ERP correlates of the bilateral redundancy gain for words. Neuropsychologia, 45(9), 2114-2124. https://doi. org/10.1016/j.neuropsychologia.2007.01.015

Mohr, B., \& Pulvermüller, F. (2002). Redundancy gains and costs in cognitive processing: Effects of short stimulus onset asynchronies. Journal of Experimental Psychology: Learning Memory and Cognition, 28(6), 1200-1223. https:// doi.org/10.1037/0278-7393.28.6.1200

Mohr, B., Pulvermüller, F., Cohen, R., \& Rockstroh, B. (2000). Interhemispheric cooperation during word processing: Evidence for callosal transfer dysfunction in schizophrenic patients. Schizophrenia Research, 46(2-3), 231-239. https://doi.org/10.1016/S0920-9964(00)00020-7

Mohr, B., Pulvermüller, F., Rayman, J., \& Zaidel, E. (1994). Interhemispheric cooperation during lexical processing is mediated by the corpus callosum: Evidence from the splitbrain. Neuroscience Letters, 181(1-2), 17-21. https://doi. org/10.1016/0304-3940(94)90550-9

Monaghan, P., \& Shillcock, R. (2008). Hemispheric dissociation and dyslexia in a computational model of reading. Brain and Language, 107(3), 185-193. https://doi.org/10.1016/J. BANDL.2007.12.005

Moore, L. H., Brown, W. S., Markee, T. E., Theberge, D. C., \& Zvi, J. C. (1995). Bimanual coordination in dyslexic adults. Neuropsychologia, 33(6), 781-793. https://doi. org/10.1016/0028-3932(95)00019-Y

Orton, S. (1927). Studies in stuttering: Introduction. Archives of Neurology \& Psychiatry, 18(5), 671-672. https://doi. org/10.1001/archneurpsyc.1927.02210050003001

Paul, L. K. (2011). Developmental malformation of the corpus callosum: A review of typical callosal development and examples of developmental disorders with callosal involvement. Journal of Neurodevelopmental Disorders, 3(1), 327. https://doi.org/10.1007/s11689-010-9059-y

Price, C. J., \& Devlin, J. T. (2011). The interactive account of ventral occipitotemporal contributions to reading. Trends in Cognitive Sciences, 15(6), 246-253. https://doi. org/10.1016/j.tics.2011.04.001

R Core Team. (2019). $R$ : A language and environment for statistical computing. Vienna, Austria: R Foundation for Statistical Computing. https://www.r-project.org

Snowling, M. J. (2005). The science of dyslexia: A review of contemporary approaches. In M. Turner \& J. Rack (Eds.),
The study of dyslexia (pp. 77-90). Springer. https://doi. org/10.1007/0-306-48534-6_4

Thomas, D. R., \& Zumbo, B. D. (2012). Difference scores from the point of view of reliability and repeated-measures ANOVA: In defense of difference scores for data analysis. Educational and Psychological Measurement, 72(1), 37-43. https://doi.org/10.1177/0013164411409929

Torgesen, J., Wagner, R., \& Rashotte, C. (1999). Test of Word Reading Efficiency (TOWRE). Pearson Assessment.

Valdois, S., Bosse, M. L., \& Tainturier, M. J. (2004). The cognitive deficits responsible for developmental dyslexia: Review of evidence for a selective visual attentional disorder. Dyslexia, 10(4), 339-363. https://doi.org/10.1002/dys.284

van der Haegen, L., Cai, Q., Seurinck, R., \& Brysbaert, M. (2011). Further fMRI validation of the visual half field technique as an indicator of language laterality: A large-group analysis. Neuropsychologia, 49(10), 2879-2888. https://doi. org/10.1016/j.neuropsychologia.2011.06.014

van der Haegen, L., Drieghe, D., \& Brysbaert, M. (2010). The split fovea theory and the Leicester critique: What do the data say? Neuropsychologia, 48(1), 96-106. https://doi. org/10.1016/j.neuropsychologia.2009.08.014

van der Knaap, L. J., \& van der Ham, I. J. M. (2011). How does the corpus callosum mediate interhemispheric transfer? A review. Behavioural Brain Research, 223(1), 211-221. https://doi.org/10.1016/J.BBR.2011.04.018

Vidyasagar, T. R., \& Pammer, K. (1999). Impaired visual search in dyslexia relates to the role of the magnocellular pathway in attention. NeuroReport, 10(6), 1283-1287. https://doi. org/10.1097/00001756-199904260-00024

Voyer, D. (1998). On the reliability and validity of noninvasive laterality measures. Brain and Cognition, 36(2), 209-236. https://doi.org/10.1006/brcg.1997.0953

Weissman, D. H., \& Banich, M. (2000). The cerebral hemisphere cooperate to perform complex but not simple task. Neuropsychology, 14(1), 41-59. https://doi. org/10.1037//0894-4105.14.1.41

Whitney, C. (2001). How the brain encodes the order of letters in a printed word: The SERIOL model and selective literature review. Psychonomic Bulletin and Review, 8(2), 221-243. https://doi.org/10.3758/BF03196158

Whitney, C., \& Cornelissen, P. (2005). Letter-position encoding and dyslexia. Journal of Research in Reading, 28, 274-301. https://doi.org/10.1111/j.1467-9817.2005.00270.x

Zaidel, E., Clarke, J., \& Suyenobu, B. (1990). Hemispheric independence: A paradigm case for cognitive neuroscience. In A. B. Scheibel \& A. F. Wechsler (Eds.), Neurobiology of higher cognitive functions (pp. 297355). Guilford Press. 